\title{
Growth inhibition and compensation in response to neonatal hypoxia in rats
}

\author{
Shlomit Radom-Aizik', Frank P. Zaldivar' ${ }^{1}$, Dwight M. Nance', Fadia Haddad ${ }^{1,2}$, Dan M. Cooper ${ }^{1}$ and Gregory R. Adams ${ }^{2}$
}

BACKGROUND: Hypoxia $(\mathrm{Hx})$ is an important disease mechanism in prematurity, childhood asthma, and obesity. In children, $\mathrm{Hx}$ results in chronic inflammation.

METHODS: We investigated the effects of $\mathrm{Hx}\left(12 \% \mathrm{O}_{2}\right)$ during postnatal days 2-20 in rats. Control groups were normoxic control (Nc), and normoxic growth restricted (Gr) (14-pup litters).

RESULTS: The Hx-exposed and Gr rats had similar decreases in growth. Hx increased plasma tumor necrosis factor- $\alpha$ (TNF- $\alpha$ ) and interleukin 6 (IL-6) levels and decreased insulin-like growth factor 1 (IGF-I) and vascular endothelial growth factor (VEGF) levels. Hx resulted in hypertrophy of the right ventricle (RV) but disproportionate decrements in limb skeletal muscle (SM) growth. miR-206 was depressed in the hypertrophied RV of Hx rats but was increased in growth-retarded SM. Hx resulted in decreased RV messenger RNA (mRNA) level for myostatin but had no effect on SM myostatin. The mRNA for Hx-sensitive factors such as hypoxia inducible factor- $1 \alpha$ (HIF-1 $\alpha$ ) was depressed in the RV of Hx rats, suggesting negative feedback.

CONCLUSION: The results indicate that $\mathrm{Hx}$ induces a proinflammatory state that depresses growth-regulating mechanisms and that tissues critical for survival, such as the heart, can escape from this general regulatory program to sustain life. This study identifies accessible biomarkers for evaluating the impact of interventions designed to mitigate the long-term deleterious consequences of $\mathrm{Hx}$ that all too often occur in babies born prematurely.

$\mathbf{T}$ here are emerging data supporting the concept of critical periods of growth during which relatively brief physiologic perturbations can lead to detrimental functional, genomic, and even epigenetic changes in affected tissues (1). In this context, premature newborns are particularly vulnerable because they are developing rapidly and because many premature newborns are exposed to profound environmental stresses such as hypoxia $(\mathrm{Hx})$ (2). Not surprisingly, premature babies are at high risk of developing growth and body composition abnormalities later in life, including failure to thrive, obesity, and osteopenia $(3,4)$.

A remarkable feature of $\mathrm{Hx}$-associated growth retardation early in life is that certain tissues, such as the heart, actually increase in size relative to body mass even in the context of overall reductions in somatic growth (5). This seeming disparity in individual tissue responses provides an opportunity to examine key regulatory mediators of simultaneous growth inhibition and compensation. By understanding the mechanisms through which Hx early in life alters growth, we might identify potential targets that could improve outcomes at critical periods of growth and development in premature babies.

The goal of this study was to test the hypotheses that (i) exposure to Hx early in life will alter the expression level of selected genes that are involved in growth and inflammatory processes in heart and skeletal muscles (SMs) of neonatal rats and (ii) the altered expression pattern will differ between heart and SM. As shown in the Results section, we focused on selected genes as well as growth and inflammatory mediators known to play a role in the regulation of heart and on $\mathrm{SM}$ as well as key microRNAs; the latter are increasingly demonstrated to play a role in gene regulation in muscle (6). We measured key anabolic mediators and inflammatory cytokines because there is evidence from this and other laboratories that growth in early life is regulated in part through the balance of growth and inflammatory mediators, the latter known to be stimulated by $\mathrm{Hx}(7,8)$. Finally, given the increasing data that gender influences gene expression responses to physiological perturbations very early in life, we hypothesized that the phenotypic, genomic, and mediator response to $\mathrm{Hx}$ would be modified by gender in neonatal rats.

\section{RESULTS}

Body Weight, Muscle Mass and Phenotype, and Organ Mass

Total body mass. At $21 \mathrm{~d}$ of age, the body mass of $\mathrm{Hx}$-exposed pups was significantly smaller than the control animals and similar to that of the growth restricted (Gr) group (Figure 1a). The growth deficits imposed either by $\mathrm{Hx}$ or large litter size appeared to be more pronounced in the male pups (Figure 1a).

Skeletal muscle. The relative mass of mixed fiber-type locomotor SMs such as the plantaris and the medial gastrocnemius (MG) were significantly depressed to a similar extent in both male and female Hx rats (Figure $\mathbf{1 b}$ and data not shown).

Right ventricle. Hx but not Gr rats experienced a remarkable adaptation in the heart in which the relative ( $\mathrm{mg} / \mathrm{g}$ body) mass of the right ventricle (RV) was increased approximately fivefold (Figure 2a). The DNA content of the RV was increased 
a

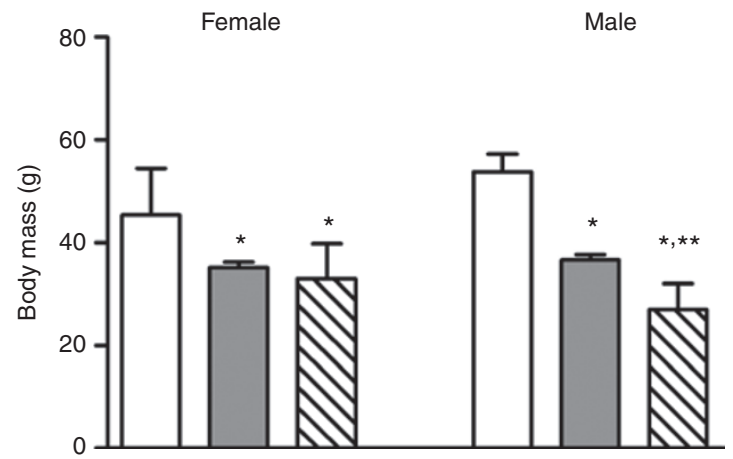

b

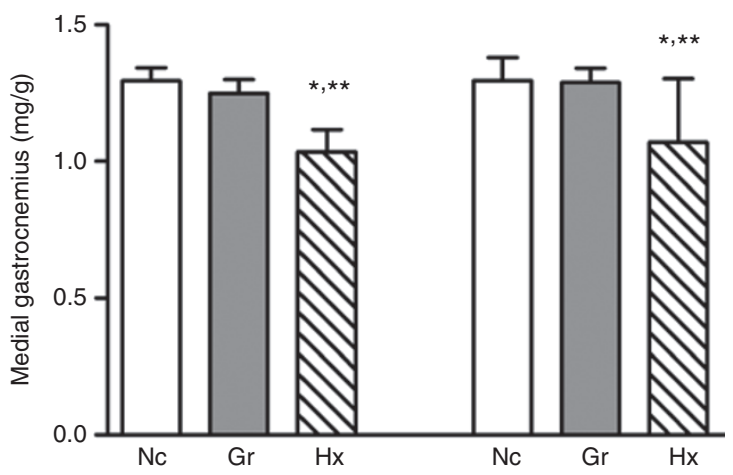

Figure 1. Effects of hypoxia and litter size on body and muscle mass. (a) Both large litter size in normoxia (growth restricted, Gr; gray column) and exposure to hypoxia $(\mathrm{Hx}$; hatched column) (litter size $=4$ ) resulted in depressed body mass relative to pups maintained in normoxia ( $\mathrm{Nc}$; white column) (litter size $=4$ ) at $21 \mathrm{~d}$ postpartum in rats. (b) The relative mass of the leg muscle medial gastrocnemius and the concentration of RNA in that muscle were depressed at $21 \mathrm{~d}$ postpartum in the $\mathrm{Hx}$ neonates. The left three columns are data from the female rats, and the right three columns are data from the male rats. $n \geq 7 ;{ }^{*} P<0.05 \mathrm{vs}$. Nc; ${ }^{* *} P<0.05 \mathrm{vs}$. Gr. approximately twofold in the Hx rats (Figure $2 \mathbf{b}$ ), whereas the DNA concentration was not different from the normoxic control (Nc) group (Table 1). In Gr rats, the DNA concentration of the RV was elevated $\sim 30 \%$ relative to that of the control and Hx-exposed animals (Table 1). The total- and myofibrillar-protein content of the RVs of Hx rats was increased approximately twofold (Figure $2 \mathrm{c}$ and Table 1). However, the concentration of total protein in the RV was similar in all groups (Supplementary Table S1 online). In contrast to content, the myofibrillar protein concentration in the RV from female $\mathrm{Hx}$ rats was $14 \%$ lower $(P<0.05)$ than in the Nc group (Table 1$)$.

As part of the adaptation to $\mathrm{Hx}$, the $\mathrm{RV}$ myosin heavy chain (MHC) phenotype of the Hx-exposed rats was shifted toward substantial expression of the $\beta$-MHC isoform (Figure 2d), reflecting a shift to a metabolically more economical phenotype (9). There were concomitant decreases $(P<0.05)$ in the expression of the $\alpha$-MHC isoform (Table 1 ).

Left ventricle. As with the RV, relative left ventricle (LV) mass was significantly increased (approximately twofold) in Hx rats relative to Nc or Gr rats (Table 2). The LV mass of the Gr rats was appropriate to the size of the animal, i.e., relative mass was not different from that of Nc. The much smaller absolute size of the Gr LV was reflected in the lower protein and DNA contents (Table 2). Relative to controls, the total protein, myofibrillar protein, and DNA content of the LV were elevated in the female Hx rats but not the males (Table 2). In both genders, the protein and DNA contents of the Hx LV were greater than those of the diet-restricted rats, reflecting the increased size of this organ.

In contrast to the content values, the concentrations of protein and myofibrillar protein were similar across groups and
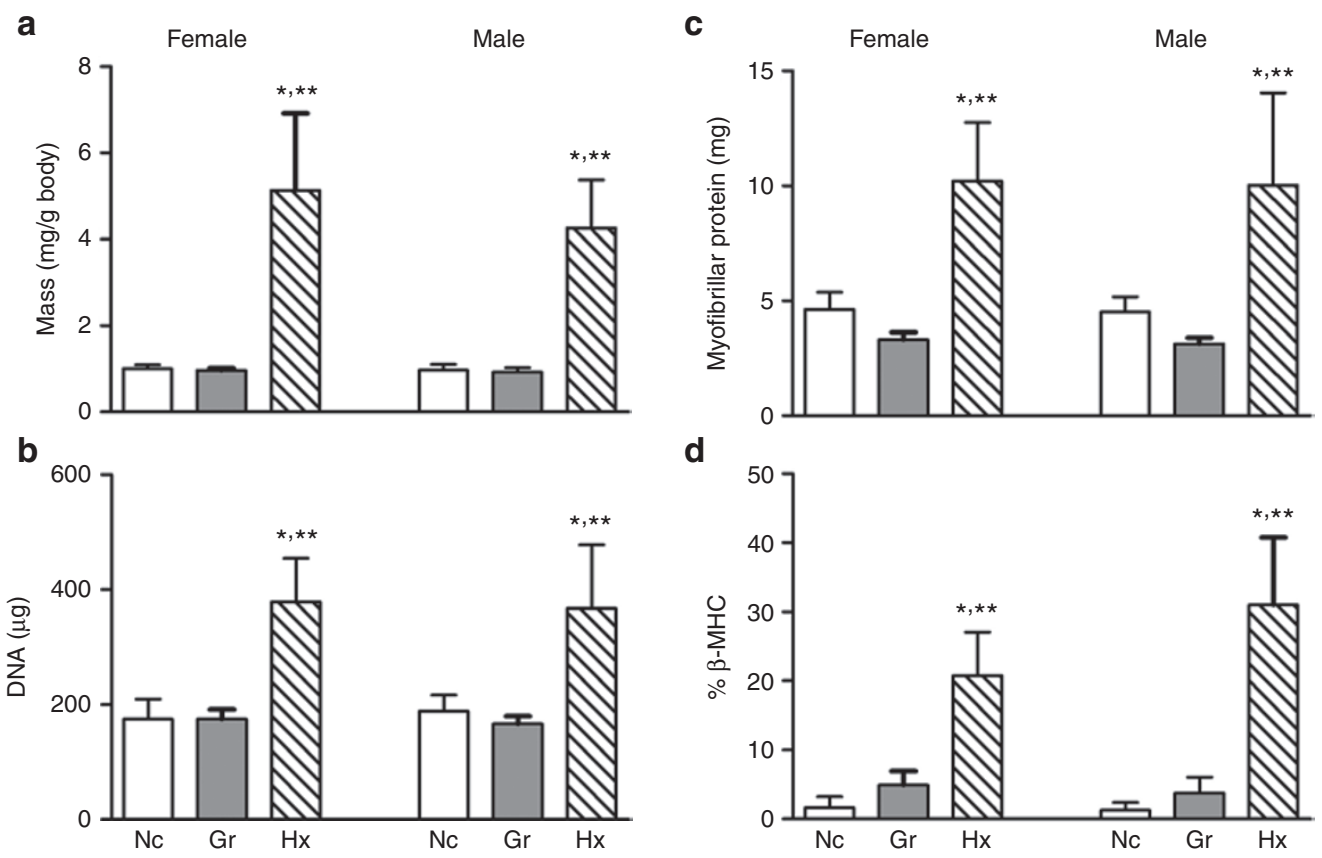

Figure 2. Effects of hypoxia and litter size on the right ventricle. The right ventricular (a) mass, (b) DNA content, (c) myofibrillar protein content, and (d) $\beta$-myosin heavy chain percent were dramatically altered to support the increased demands of the hypoxic treatment at $21 \mathrm{~d}$ postpartum. $n \geq 7$. ${ }^{*} P<0.05$ vs. Nc; ${ }^{* *} P<0.05$ vs. Gr. Gr, growth restricted; $M H C$, myosin heavy chain; Nc, normoxic control. 
genders in the LV (Supplementary Table S2 online). Similar to the RV, the concentration of DNA in the LV was slightly greater in Gr rats (Supplementary Table S2 online). However, in the LV, significant differences were only seen in the female Gr rats.

The LV of the Hx rats demonstrated a shift in $\beta$-MHC phenotype that was qualitatively similar to that seen in the RV (Table 2). There was a complementary decrease $(P<0.05)$ in the expression of the $\alpha$-MHC isoform in the LV (Table 2).

\section{Growth Factors, Inflammatory Mediators, and Leukocytes}

Plasma estradiol concentration was lower in Nc males as compared with Nc females (e.g., Nc: $30 \pm 3$ vs. $40 \pm 4$ pg/ml; $P=0.04$, Student's $t$-test). Estradiol appeared to be or was significantly elevated in the Hx and Gr males (Table 3). There were no differences in plasma testosterone between the Nc male and female animals (Table 3). Plasma testosterone was significantly depressed in the female $\mathrm{Hx}$ rats relative to the $\mathrm{Nc}$ females (Table 3 ).
Circulating levels of insulin-like growth factor 1 (IGF-I) were lower than those of the control in both $\mathrm{Hx}$ and $\mathrm{Gr}$ animals (Figure 3a). Further, IGF-I in Hx rats was significantly lower than in Gr animals (Figure 3a). Vascular endothelial growth factor (VEGF) and growth hormone levels were reduced in the $\mathrm{Hx}$-exposed pups but not in diet-restricted pups (Figure 3b,c).

In $\mathrm{Hx}$ rats, the concentrations of monocytes and neutrophils were elevated in males but not females (Table 3 ). Tumor necrosis factor (TNF)- $\alpha$ and interleukin 6 (IL)-6 levels were significantly elevated in the Hx-exposed pups (Table 3). Hemoglobin concentrations were elevated in both the $\mathrm{Hx}$-exposed and diet-restricted pups, with greater changes seen in the Hx group (Table 3 ).

\section{Gene Expression}

Heart. In the RV, the levels of several Hx-sensitive messenger RNAs (mRNAs) were significantly altered. The Hx animals experienced decreases in hypoxia inducible factor- $1 \alpha$ $(H I F-1 \alpha)$ and hypoxia up-regulated 1 (HYOU1) in both

Table 1. Treatment effects in the right ventricle

\begin{tabular}{|c|c|c|c|c|c|c|}
\hline & \multicolumn{3}{|c|}{ Female } & \multicolumn{3}{|c|}{ Male } \\
\hline & $\mathrm{Nc}$ & Gr & $\mathrm{Hx}$ & $\mathrm{Nc}$ & Gr & $\mathrm{Hx}$ \\
\hline Protein content (mg) & $10.1 \pm 0.5$ & $7.8 \pm 0.2$ & $26.8 \pm 2.8^{*, * *}$ & $12.2 \pm 0.8$ & $8.8 \pm 0.4$ & $27.6 \pm 4.5^{*, * *}$ \\
\hline Myofibrillar protein conc. (mg/g) & $93.0 \pm 2.6$ & $89.7 \pm 2.8$ & $79.9 \pm 2.6^{*}$ & $81.7 \pm 4.1$ & $85.7 \pm 2.2$ & $81.1 \pm 3.1$ \\
\hline DNA conc. (mg/g) & $2.83 \pm 0.16$ & $3.80 \pm 0.14$ & $2.47 \pm 0.16$ & $2.73 \pm 0.06$ & $3.650 \pm 07$ & $2.57 \pm 0.19$ \\
\hline RNA content $(\mu \mathrm{g})$ & $134 \pm 8$ & $83 \pm 4$ & $359 \pm 54^{*, * *}$ & $132 \pm 5$ & $93 \pm 3$ & $302 \pm 54^{*, * *}$ \\
\hline$\alpha-\mathrm{MHC}(\%$ total) & $98.4 \pm 0.7$ & $95.1 \pm 0.9$ & $79.2 \pm 2.7^{*, * *}$ & $98.8 \pm 0.5$ & $96.3 \pm 1.0$ & $69.0 \pm 3.9^{*, * *}$ \\
\hline miR-133a (AU) & $1.30 \pm 0.17$ & $1.88 \pm 0.16$ & $1.15 \pm 0.23^{* *}$ & $1.01 \pm 0.06$ & $1.12 \pm 0.12$ & $0.98 \pm 0.11$ \\
\hline
\end{tabular}

Table 2. Treatment effects in the left ventricle

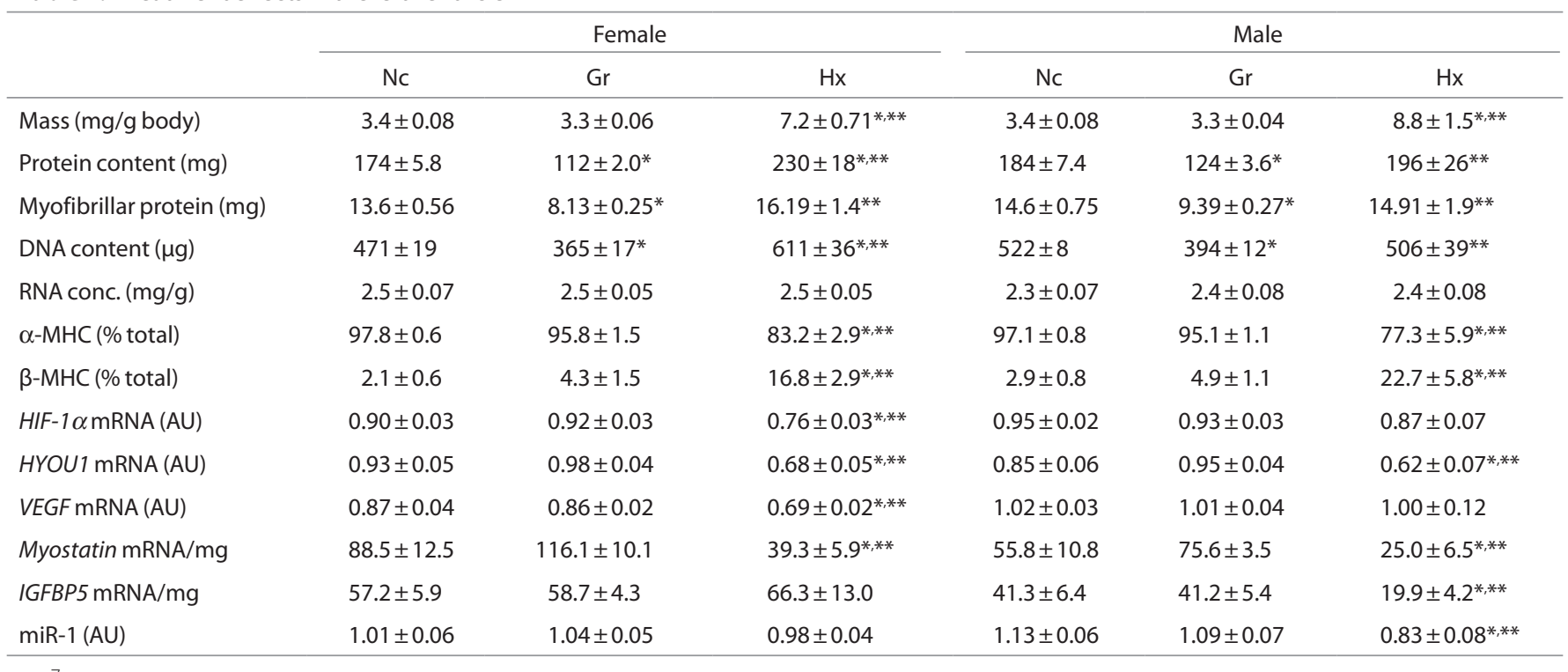

$n \geq 7$

AU, arbitrary units; conc., concentration; Gr, growth restricted; HIF-1 $\alpha$, Hx inducible factor- $1 \alpha$; Hx, hypoxia; / GFBP5, insulin-like growth factor binding protein-5; MHC, myosin heavy chain; HYOU1, Hx upregulated-1; MHC, myosin heavy chain; Nc, normoxic control; VEGF, vascular endothelial growth factor.

$* P<0.05$ vs. Nc; ${ }^{* *} P<0.05$ vs. Gr. 
Table 3. Treatment effects on plasma

\begin{tabular}{|c|c|c|c|c|c|c|}
\hline & \multicolumn{3}{|c|}{ Female } & \multicolumn{3}{|c|}{ Male } \\
\hline & Nc & $\mathrm{Gr}$ & $\mathrm{Hx}$ & $\mathrm{Nc}$ & $\mathrm{Gr}$ & $\mathrm{Hx}$ \\
\hline Monocytes $(\mathrm{K} / \mu \mathrm{l})$ & $0.38 \pm 0.08$ & $0.20 \pm .011$ & $0.23 \pm 0.06$ & $0.34 \pm 0.11$ & $0.27 \pm 0.11$ & $0.90 \pm 0.30^{* * * *}$ \\
\hline TNF- $\alpha(p g / m l)$ & $2.56 \pm 0.23$ & $3.05 \pm 0.13$ & $3.86 \pm 0.27^{*}$ & $2.68 \pm 0.15$ & $2.91 \pm 0.10$ & $3.96 \pm 0.25^{* * * *}$ \\
\hline IL-6 (pg/ml) & $14.0 \pm 1.5$ & $16.0 \pm 2.8$ & $27.2 \pm 2.0^{*, * *}$ & $13.5 \pm 1.5$ & $18.7 \pm 1.6$ & $30.2 \pm 3.7^{*, * *}$ \\
\hline Testosterone $(\mathrm{ng} / \mathrm{ml})$ & $1.58 \pm 0.22$ & $1.07 \pm 0.11$ & $0.82 \pm 0.09^{*}$ & $1.52 \pm 0.14$ & $1.12 \pm 0.08$ & $0.95 \pm 0.35$ \\
\hline Estradiol (pg/ml) & $40.3 \pm 3.8$ & $37.0 \pm 2.7$ & $40.0 \pm 8.0$ & $30.2 \pm 2.5$ & $42.1 \pm 2.4^{*}$ & $47.4 \pm 12.0$ \\
\hline
\end{tabular}

$n \geq 7$.

Gr, growth restricted; IL-6, interleukin-6; Nc, normoxic control; TNF- $\alpha$, tumor necrosis factor- $\alpha$.

${ }^{*} P<0.05$ vs. Nc; ${ }^{* *} P<0.05$ vs. Gr.

a

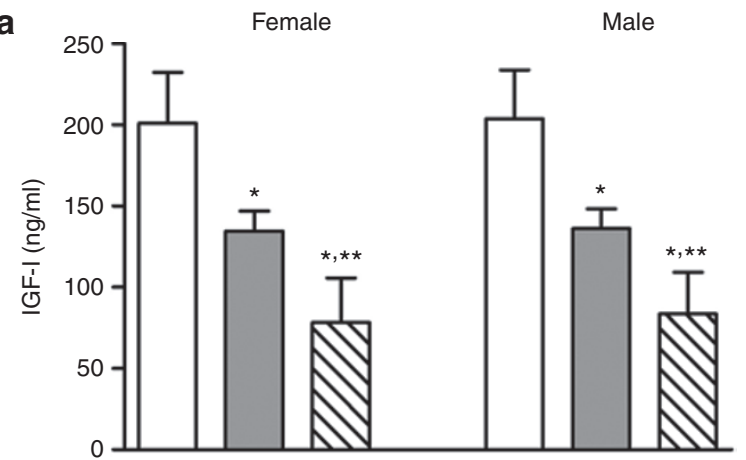

b

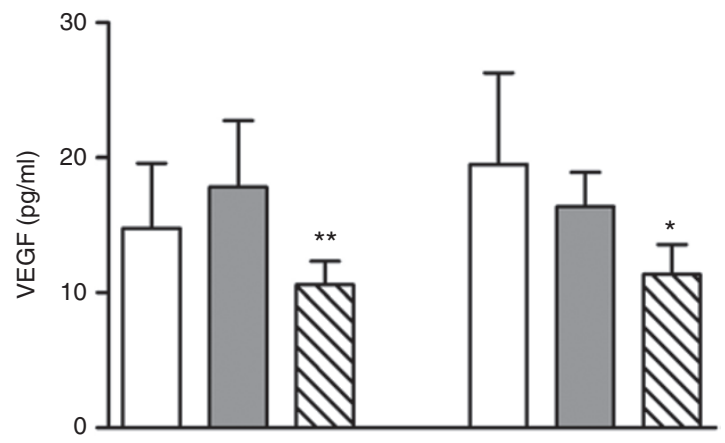

C

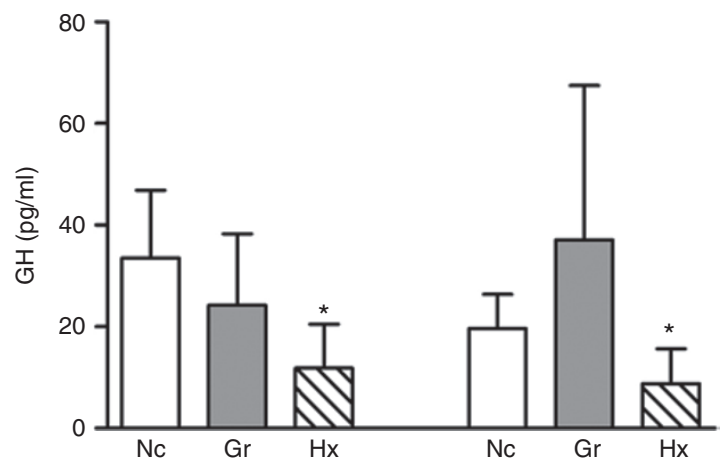

Figure 3. Effects of hypoxia and litter size on circulating growth mediators. At $21 \mathrm{~d}$ postpartum, plasma concentrations of (a) IGF-I were depressed in both the $\mathrm{Gr}$ and $\mathrm{Hx}$ neonates. Plasma concentrations of (b) VEGF and (c) GH were depressed only in the Hx groups. $n \geq 7,{ }^{*} P<0.05$ vs. Nc; ${ }^{* *} P<0.05$ vs. Gr. GH, growth hormone; Gr, growth restricted; $\mathrm{Hx}$, hypoxia; IGF-I, insulin-like growth factor-1; Nc, normoxic control; VEGF, vascular endothelial growth factor. genders (Figure 4a,b) and decreased VEGF in the females (Figure 4c). However, the mRNA for platelet-derived growth factor receptor- $\beta$, known to be critical for angiogenic adaptation in overloaded hearts, was increased in the RV of $\mathrm{Hx}$ animals (Figure 4d).

$\mathrm{Hx}$ also affected the mRNA levels of several growth-related factors in the heart. The levels of mRNA for myostatin, a powerful negative regulator of muscle growth, and IGF-I binding protein 5 (IGFBP-5), a binding protein sensitive to IGF-I levels, were depressed in both the RV (Figure 5a,b) and the LV (Table 2) of Hx animals. No differences were observed in the levels of mRNA for IGFBP4, Cyclin D1, and Nos3 (Supplementary Table S1 online).

In the LV, the levels of several $\mathrm{Hx}$-sensitive mRNAs were significantly altered, primarily in the female rats. The female $\mathrm{Hx}$ animals experienced decreases in HIF-1 $\alpha$ and VEGF mRNA concentrations, and HYOU1 mRNA concentration was decreased $\sim 26 \%$ in both genders (Table 2). The concentration of platelet-derived growth factor receptor- $\beta$ mRNA was not different across treatments (Supplementary Table S2 online).

Skeletal muscle. The mRNA concentrations for Nos3, a proangiogenic regulator (10), and IGFBP4 were significantly depressed in the MG muscles from Hx rats of both genders (Figure 5c,d). In male Hx rats, the mRNA levels for IGFBP5 were also significantly lower than those found in the $\mathrm{Nc}$ and Gr animals (Table 4). The mRNA of muscle ring finger-1 and atrogin-1, muscle-specific E3 ligases often associated with muscle atrophy (11), were not significantly different between groups (Supplementary Table S3 online). Interestingly, the mRNA for myostatin was not different across groups (Table 4). No treatment-associated changes were seen in MHC isoform expression (data not shown). In the MG muscle, the mRNA concentrations for HIF-1 $\alpha$, VEGF, HYOU1, and platelet-derived growth factor receptor- $\beta$ were similar across groups (Supplementary Table S3 online).

\section{MicroRNAs}

Levels of microRNAs known to be important regulators of gene expression in the heart were affected by exposure to 
a

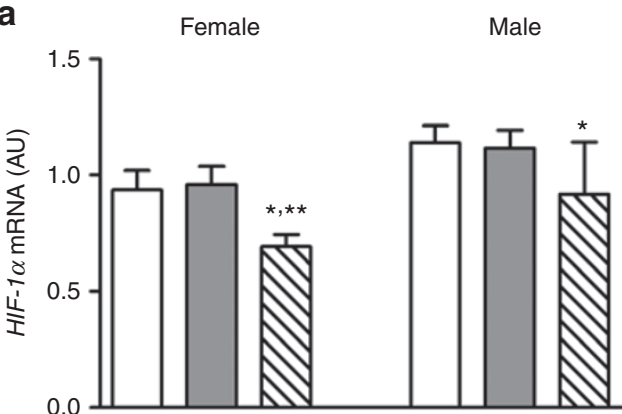

b

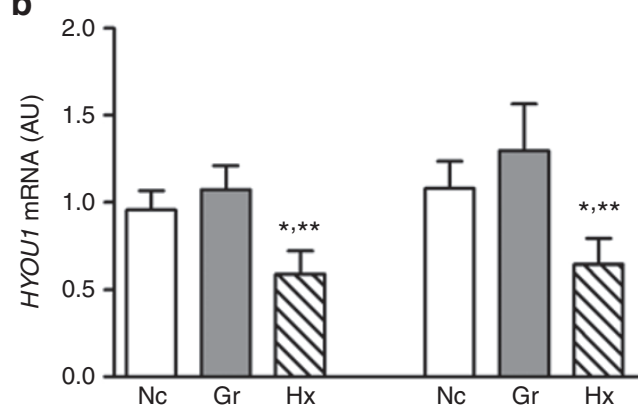

C

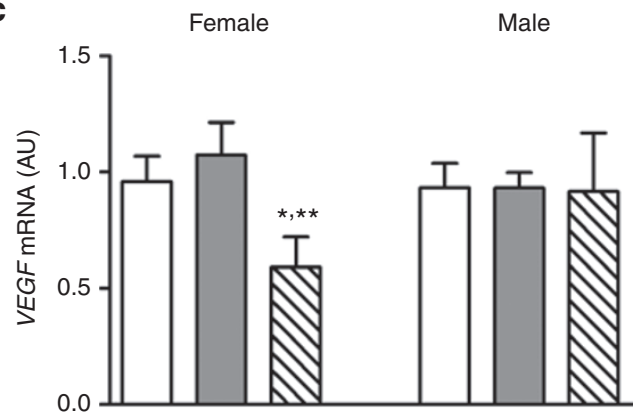

d

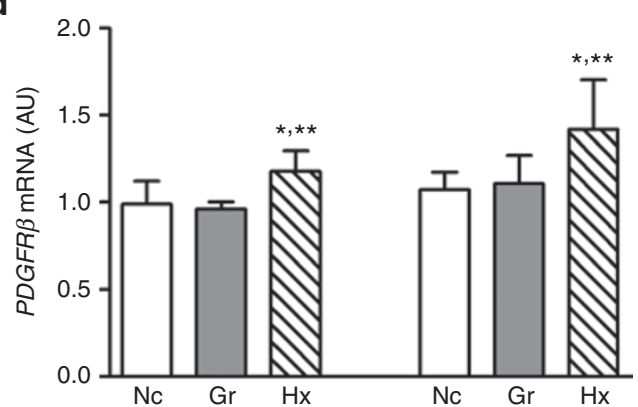

Figure 4. Effects of hypoxia and litter size on hypoxia-sensitive mRNA in the right ventricle. Abundance of mRNA for (a) HIF-1 $\alpha$, (b) HYOU1, (c) VEGF, and (d) PDGFR $\beta$ was altered in the right ventricle at $21 \mathrm{~d}$ postpartum. An exception to this was seen for VEGF in the Hx male neonates. $n \geq 7,{ }^{*} P<0.05$ vs. $\mathrm{Nc}$; ${ }^{* *} P<0.05$ vs. Gr. AU, arbitrary units; Gr, growth restricted; HIF-1 $\alpha$, hypoxia inducible factor-1 $\alpha ;$ HYOU1, hypoxia upregulated-1; Nc, normoxic control; $P D G F R \beta$, platelet-derived growth factor receptor- $\beta$; VEGF, vascular endothelial growth factor.
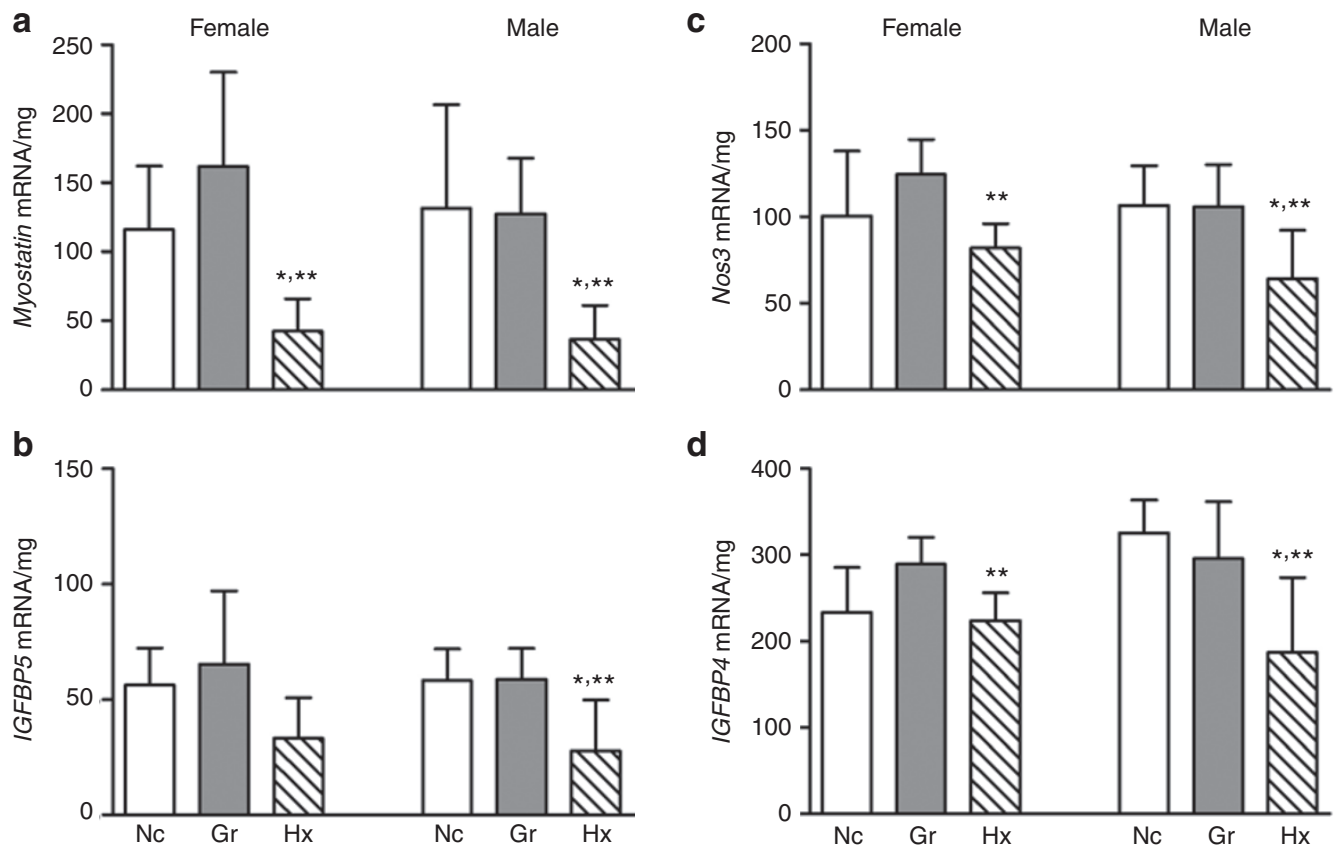

Figure 5. Effects of hypoxia and litter size on growth-related mRNA in the right ventricle and skeletal muscle. The abundance of mRNA for (a) myostatin and (b) IGFBP5 was significantly depressed in the right ventricles of the Hx neonates at $21 \mathrm{~d}$ postpartum. The abundance of mRNA for (c) Nos 3 and (d) IGFBP4 was depressed in the MG muscle. $n \geq 7,{ }^{*} P<0.05$ vs. Nc; ${ }^{*} P<0.05$ vs. Gr. Gr, growth restricted; Hx, hypoxia; IGFBP, insulin-like growth factor binding protein; MG, medial gastrocnemius; Nc, normoxic control.

Hx. In the RV, miR-206 was depressed in both genders, as was miR-1 in male Hx rats (Figure 6a,b). The expression of miR-133a was increased in the RV of female Gr rats relative to the Hx group (data not shown); no other differences were observed in the RV. Only one significant change in
miR abundance was observed in the LV. The expression of miR-1 decreased $27 \%$ in the LV of male but not female $\mathrm{Hx}$ rats (Table 2).

Analysis of candidate microRNAs commonly reported to be important in SM demonstrated various responses that were often 


\section{Articles $\mid$ Radom-Aiziketal.}

Table 4. Treatment effects in the medial gastrocnemius muscle

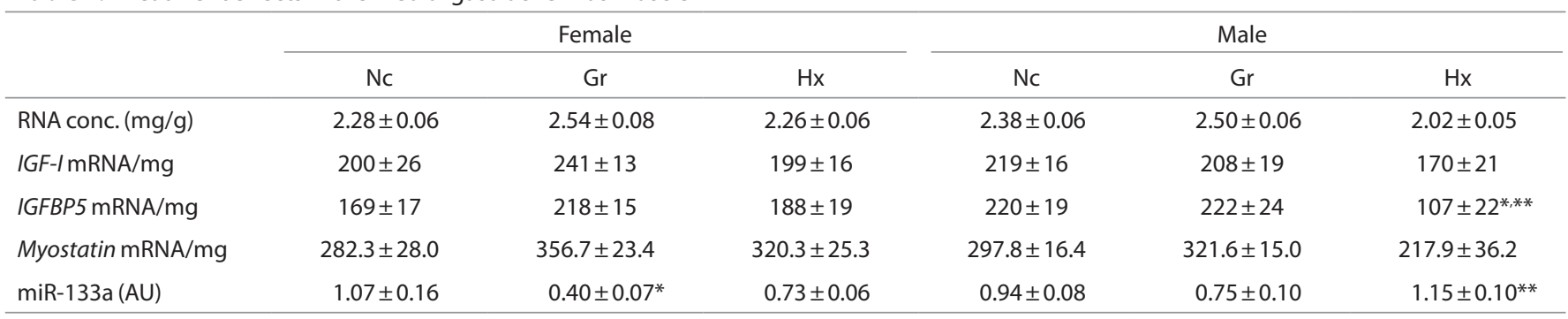

$n \geq 7$.

$\mathrm{AU}$, arbitrary units; conc., concentration; Gr, growth restricted; HX, hypoxia; /GF-1, insulin-like growth factor-1; /GFBP5, insulin-like growth factor binding protein-5; Nc, normoxic control. ${ }^{*} P<0.05$ vs. Nc; ${ }^{* * P}<0.05$ vs. Gr.
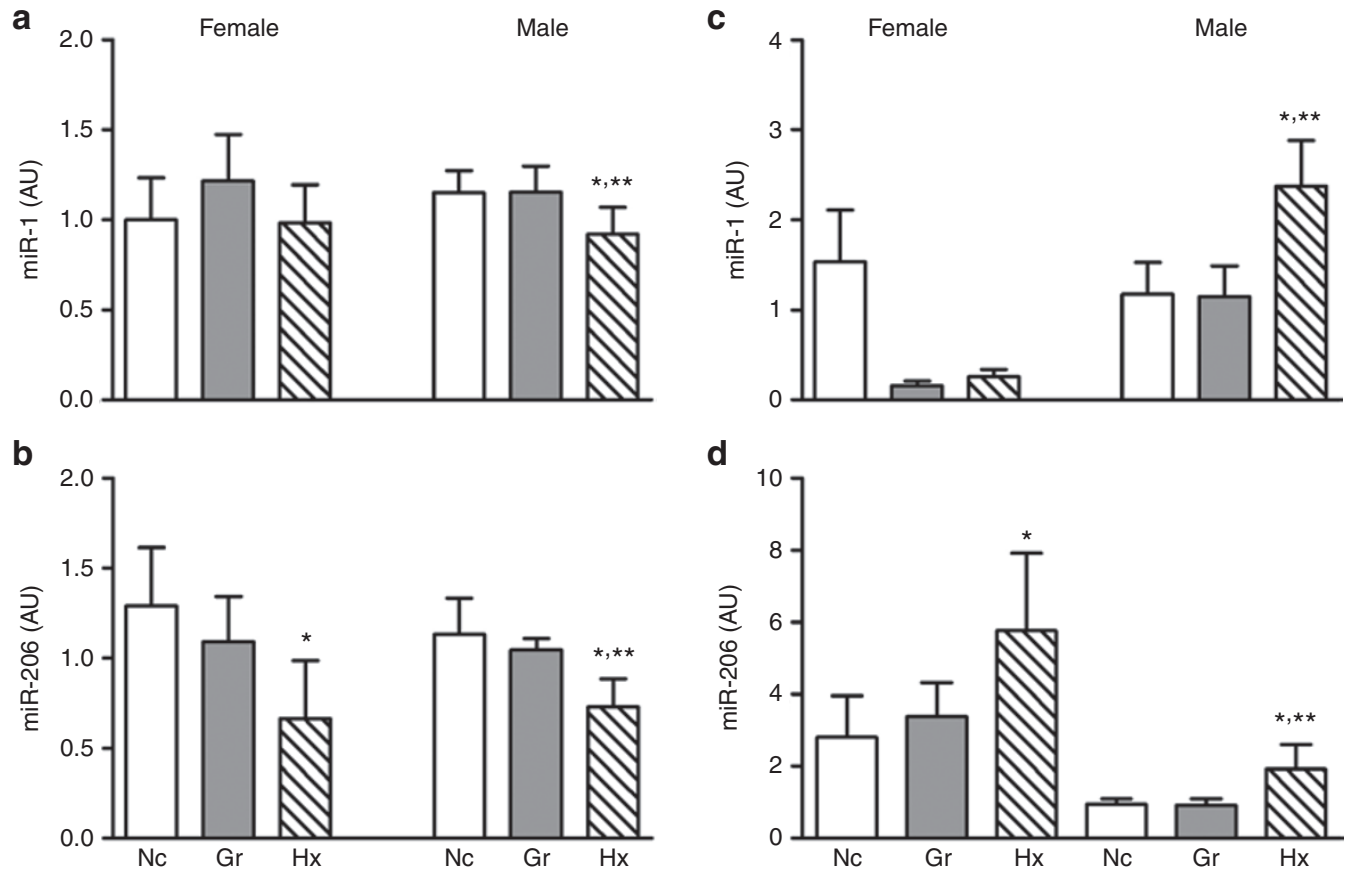

Figure 6. Effects of hypoxia and litter size on microRNA expression in heart and skeletal muscle. At $21 \mathrm{~d}$ postpartum, the abundance of (a) miR-1 was depressed in the RV of male neonates. At this time point, the abundance of (b) miR-206 was depressed in the RV of both genders. In MG muscle, miR-1 (c) was depressed in both the $\mathrm{Gr}$ and $\mathrm{Hx}$ female neonates. In contrast, the miR-206 (d) was increased in both genders of Hx neonates. $n \geq 7$; ${ }^{*} P<0.05$ vs. $\mathrm{Nc}$; ${ }^{*} P<0.05$ vs. Gr. AU, arbitrary units; Gr, growth restricted; $\mathrm{Hx}$, hypoxia; MG, medial gastrocnemius; Nc, normoxic control; RV, right ventricle.

gender specific. MG muscle miR-1 was significantly elevated in male $\mathrm{Hx}$ rats, whereas miR-1 was repressed in females in the $\mathrm{Hx}$ and Gr groups (Figure 6c). The expression level of miR-206 was elevated in the MG muscles of $\mathrm{Hx}$ rats in both genders (Figure 6b). miR-133a was repressed in female Gr rats (Table 4).

\section{DISCUSSION}

In this study, we succeeded in comparing two types of somatic growth inhibition, one with $\mathrm{Hx}$ and one with large litter size, in neonatal rats. The comparison permitted us to shed new light on molecular mechanisms of both growth stimulation, in the hearts of Hx-exposed rats, and growth inhibition in the SMs. We identified, for the first time, potential $\mathrm{Hx}$-specific gene mediators of growth such as decreased cardiac myostatin and IGFBP5 expression in Hx-exposed neonatal rats. We identified the potential pivotal role played by the microRNA miR-206 as an $\mathrm{Hx}$-sensitive regulator of growth in cardiac muscle in neonatal rats. Our studies further elucidate the unique role that Hx plays in promoting inflammatory mediators, a phenomenon that is potentially linked to the long-term consequences of Hx early in life. Finally, our studies suggest that the impact of moderate $\mathrm{Hx}$ early in life may alter growth to a greater extent through secondary mediators, such as inflammatory cytokines, than through direct effects of $\mathrm{Hx}$ on known regulatory genes, such as HYOU1, HIF-1 $\alpha$, and VEGF. These results are encouraging, given that we previously found evidence that such secondary effects (such as inflammation) can be attenuated by physical activity $(12,13)$.

It is conceivable that the paradoxical growth effects that we observed, namely, a robust increase in relative heart-muscle size in the Hx-exposed rats despite their smaller size, resulted from somewhat antagonistic simultaneous effects of $\mathrm{Hx}$ 
exposure and increased muscle work. Even under the conditions of moderate Hx imposed in the current study, cardiac work would be increased: in the right heart, likely caused by Hx-induced pulmonary vasoconstriction (14); in the left heart, through the adaptive response of increasing systemic cardiac output to maintain tissue oxygen delivery in light of reduced arterial blood oxygenation. SM, in contrast, would not be similarly stimulated by $\mathrm{Hx}$.

In the heart, an important finding was the decreased expression of VEGF, either as protein present (circulation) or message (tissues), associated specifically with the $\mathrm{Hx}$ exposure. VEGF plays a key role in the angiogenesis that occurs in SM in response to exercise training (15) and heart muscle in response to loading and $\mathrm{Hx}$ (16). VEGF gene expression is sensitive to Hx through mediators such as HIF-1 $\alpha$ (15). In the current study, both the circulating and tissue levels of message for $V E G F$ and/or VEGF regulatory mediators (e.g., HIF-1 $\alpha$ and $I G F-I)$ (17) were actually depressed, thereby indicating that these regulatory feedback pathways may have been invoked during Hx exposure. These data strongly suggest that in future studies functional indexes of angionesis, such as capillary density, should be examined.

This potential differential mechanism is highlighted in the comparison of skeletal and cardiac muscle. In contrast to the heart, the changes in VEGF or its regulatory genes were not influenced by $\mathrm{Hx}$ in SM. This result is similar to that reported by He et al. (18) in adult rats: Hx per se had no effect in muscle expression of HIF-1 $\alpha$ and VEGF. Interestingly, these authors found increased expression of the mRNA for these mediators in adult animals that were exposed to $\mathrm{Hx}$ in combination with exercise, suggesting that an additional stressor is necessary to induce adaptations in this tissue (18).

Developmental growth of the heart is modulated both by mechanical factors and mediators such as IGF-I (19). In the case of the IGF-I system, the results seen in the current study (Figure 3) are consistent with observations that a decrease in IGFBP5 expression may promote growth/hypertrophy (20). However, in contrast to Hx imposed in adult rats (21), the RV hypertrophy seen in the current study did not appear to be driven by an increase in the mRNA for IGF-I itself.

Similar to the heart, SM developmental growth is coordinated, in part, by circulating mediators such as IGF-I (22). In that context, the restrained growth of the leg SMs investigated was consistent with the depressed circulating levels of growth factors (Figure 3). In the current study, the mRNA for IGFPB4 was depressed by Hx in both genders. This IGF binding protein is commonly considered to function as a negative regulator of IGF-I. However, IGFBP4 gene knockout has been reported to decrease prenatal growth (23), suggesting that the decrease observed in the current study may be consonant with the observed growth inhibition.

In contrast to studies imposing $\mathrm{Hx}$ on adult rats (24), the limb SMs of neonates in this study did not demonstrate enhanced levels of myostatin mRNA, suggesting that this mediator did not participate in the Hx-induced growth retardation. Interestingly, the enhanced growth seen in the heart of
Hx-treated rats did appear to be a function, at least in part, of a decrease in myostatin mRNA (Figure 5, Table 2).

An additional mechanistic contrast between the response of the neonatal heart and SMs to Hx is seen in the levels of mRNA for angiogenic regulators. As noted above, in the heart, VEGF mRNA levels were depressed in the Hx females (Figure 4, Table 2) whereas Nos3 mRNA was unchanged (Supplementary Table S1 online). In SM, VEGF mRNA was unaffected by Hx (Supplementary Table S3 online), whereas Nos3 mRNA levels were depressed in both females and males (Figure 5).

With regard to miR expression, the most interesting and clear-cut result was seen for miR-206. Hx but not growth restriction uniformly increased the expression of this $\mathrm{miR}$ in SM, a tissue that experienced a profound decrement in growth. In contrast, the RV, which escaped the somatic anti-growth signaling program, had decreased miR-206 expression. This result suggests that, in a developmental setting, miR-206 may be a critical regulator in striated muscle.

In SM, the upregulation of miR-206 is consistent with a decrease in satellite cell proliferation and indications of increased differentiation (25). This response would be expected to strongly limit total muscle size during development, i.e., fewer myofibers. The role of miR206 in the development of the heart is less clear. In mature animals, miR-206 expression is increased in response to injury and appears to contribute to the proliferation of progenitor cells.

In both skeletal and cardiac muscle, miR-206 appears to function via the downregulation of tissue inhibitor of metalloproteinases-3 (26). Tissue inhibitor of metalloproteinases-3 in turn appears to be a critical regulator of muscle regeneration (26). In the present study, the Hx RV demonstrated a marked decrease in miR206 expression, whereas the concentration of DNA was similar to that in RV of the Nc animals. This suggests that the downregulation of miR-206-biased cardiac adaptation toward hypertrophy and away from myogenesis, possibly as a strategy to match cardiomyocyte growth with vascularization (27).

The results from a number of studies have indicated that early-in-life Hx results in lasting changes in the cardiovascular system and metabolism $(5,28,29)$. For example, Del Duca et al. (28) reported that, in rats, $10 \mathrm{~d}$ of neonatal exposure to $\mathrm{Hx}$ resulted in differential gene expression in the LV in adulthood. In that study, genes regulating a range of functions such as apoptosis, metabolism, and vascular remodeling were affected by neonatal Hx. This research group found that the brief period of Hx exposure during this critical period of development had lasting effects on cardiomyocyte function, morphology, and viability $(28,29)$.

In the current study, exposure to Hx resulted in increased levels of circulating inflammatory mediators (Table 3) and, in the male rats, an elevation of the number of monocytes and neutrophils in the circulation (Table 3). The activation of inflammatory processes by $\mathrm{Hx}$ has been reported in a number of previous studies (30). In an interesting study using intravital microscopy, Dix et al. (31) reported that inflammation in 
microcirculatory beds was increased in response to systemic but not local Hx. Subsequent studies reported that alveolar macrophages mediate inflammatory responses in tissues remote from the lung (32). These results demonstrate that inflammatory responses to $\mathrm{Hx}$ are regulated via global mechanisms with the potential to affect all of the somatic systems.

In general, proinflammatory cytokines entrain a number of intracellular processes that would be antianabolic in muscle (33). TNF- $\alpha$ is thought to exert negative effects on cardiac muscle growth and function (33). However, some studies suggest a more complex relationship in which TNF- $\alpha$ may play a role in myogenesis following muscle injury (27).

Similar to TNF- $\alpha$, IL- 6 has been reported to play a variety of roles, both positive and negative, in cardiac pathology $(27,34)$. However, in the heart, the pathological effects of IL-6 expression are generally associated with hypertrophy.

It is well established that physiological insults, such as malnutrition or inflammation, during fetal life can adversely and profoundly affect subsequent growth and development (1). As noted above, it is also well established that exposure to $\mathrm{Hx}$ induces systemic inflammation $(31,35)$. There is some evidence that early-in-life $\mathrm{Hx}$ results in lasting changes in the cardiovascular system and metabolism $(5,28,29)$. However, little is known about the long-term effects of $\mathrm{Hx}$ and attendant inflammation experienced during critical periods of postnatal life. This is a critical gap in our knowledge base because chronic diseases of childhood such as asthma and obesity may not only impair day-to-day health in affected individuals but may adversely affect health throughout life (36). The results from the current study point to mechanisms that may mediate lifelong changes resulting from a hypoxic episode experienced during a critical period of development. Such knowledge can be used to identify accessible biomarkers for evaluating the impact of interventions designed to mitigate the long-term deleterious consequences of $\mathrm{Hx}$ that all too often occur in babies born prematurely.

\section{METHODS}

Pregnant Sprague-Dawley rats were purchased from Charles River (Wilmington, MA). Immediately postpartum, the litters were randomly cross fostered and gender balanced. At postpartum day 3, four litters were randomized to the $\mathrm{Hx}$ treatment group (Hx-exposed), culled to four pups per litter (total: eight male and eight female), and housed, with the dam, in standard cages placed in a normobaric chamber Biospherix ProOx 360 (Biospherix, Lacona, NY). The small litter size was adopted to ensure adequate nutrition and minimize the maternal stress associated with full litter size in the hypoxic environment (28). The four litters randomized to the Nc groups were also culled to four pups (total: eight male and eight female) and housed in standard cages in the same room as the chamber. The protocols used in this study were approved by the University of California Irvine Institutional Animal Care and Use Committee.

To induce $\mathrm{Hx}$, a feedback controller senses $\mathrm{O}_{2}$ levels in the chamber and feeds $\mathrm{N}_{2}$ into the chamber to maintain the preset level of $\mathrm{O}_{2}(12 \%)$. We have modeled $\mathrm{Hx}$ at an $\mathrm{FiO}_{2}$ of $12 \%$, which represents moderate hypoxic exposure, equivalent to the ambient $\mathrm{FiO}_{2}$ found at $4,000 \mathrm{~m}$ and quite compatible with life. In humans, inhalation of $12.5 \% \mathrm{O}_{2}$ has been reported to decrease systemic $\mathrm{PO}_{2}$ to $<50$ Torr (37). Using the alveolar gas equation, this exposure should produce $\sim 80-85 \%$ oxygen saturation of hemoglobin in the rat. We and others have found that this level of $\mathrm{Hx}$ is "tolerated" by the young rats (i.e., the vast majority survive) (28). The chamber used in this study allows exchange with the environment so that the pressure remains unchanged. Humidity and $\mathrm{CO}_{2}$ were monitored and regulated.

To provide a comparison group that experienced growth restriction without $\mathrm{Hx}$, three additional litters were created consisting of 14 pups per dam (total: 21 male and 21 female). This treatment is known to result in significant growth impairment (38). The diet-restricted groups were also housed in standard cages in the same room as the chamber. Previous studies indicated that $\mathrm{Hx}$ exposure is associated with some degree of reduced food intake, but not of sufficient magnitude to account for the decrements in growth seen with $\mathrm{Hx}(28)$.

\section{Tissue Collection and Analysis}

The study was terminated on day 21 postpartum. The rats were euthanized using Pentosol solution. After the induction of deep anesthesia, but before the cessation of breathing, blood was collected from the LV via the diaphragm using a heparinized syringe. The ventricles were removed and weighed. The soleus, plantaris, and MG muscles of both legs were dissected free of connective tissue and weighed. All tissues were snap-frozen and stored at $-80^{\circ} \mathrm{C}$ for later analysis.

\section{Heart and SM Protein, Myofibrillar Protein, and DNA}

Tissue samples were homogenized in $20 \mathrm{ml}$ of buffer per gram tissue. The homogenization buffer contained $250 \mathrm{mmol} / \mathrm{l}$ sucrose, 100 $\mathrm{mmol} / \mathrm{l} \mathrm{KCl}, 5 \mathrm{mmol} / \mathrm{l} \mathrm{EDTA}$, and $10 \mathrm{mmol} / \mathrm{l}$ Tris $\mathrm{HCl}$; $\mathrm{pH} 7.0$. Myofibrillar proteins were quantitatively extracted from a known volume of the total homogenate suspended into a known volume of $100 \mathrm{mmol} / \mathrm{l} \mathrm{KCl}, 10 \mathrm{mmol} / \mathrm{l}$ Tris, and $1 \mathrm{mmol} / \mathrm{l}$ EDTA; $\mathrm{pH}$ 7.4. Protein concentration in the homogenate and myofibril suspension was determined using the Biorad Protein assay with gamma globulin as a standard. Muscle protein and myofibril content were calculated on the basis of homogenized muscle piece weight and total muscle weight. Calculation of muscle total DNA concentration was based on total DNA concentration in the total homogenate and was determined by a fluorometric assay using the DNA-specific fluorescent Hoechst 33258 dye (39).

In each case, the data provided include both the content and concentration for the protein, myofibrillar protein, RNA, and DNA of the tissues examined. This was done to provide an appreciation of both the changes in size that occurred in response to the treatment (content values) and relevant concentrations so that the reader can determine whether these values remained in a normal physiological range.

\section{Complete Blood Count}

Complete blood count with differential for hemoglobin and white blood cell analysis were obtained using Hemavet 950 (Drew Scientific, Waterbury, CT).

\section{Plasma Analysis}

Levels of circulating cytokines (TNF- $\alpha$ and IL-6), growth factors (IGF-I, growth hormone, and VEGF), and sex hormones (testosterone and estradiol) were measured using commercially available enzyme-linked immunosorbent assay kits manufactured by R\&D Systems (Minneapolis, MN).

\section{RNA Analysis}

Total RNA. Total RNA was extracted from preweighed frozen tissue samples using the TRI Reagent according to the manufacturer's protocol (Molecular Research Center, Cincinnati, OH). Total RNA concentration was determined by optical density at $260 \mathrm{~nm}$. The tissue total RNA concentration was calculated on the basis of total RNA yield and the weight of the analyzed sample.

mRNA analysis. One microgram of total RNA was reverse transcribed into cDNA using the SuperScript II RT from Invitrogen (Grand Island, NY) and a mix of oligo dT and random primers.

Cardiac MHC mRNA, IGF-I, IGFBP4, IGFBP5, myostatin, muscle ring finger-1, Atrogin, Cyclin D1, and Nos3 mRNA expression levels were analyzed using an end-point reverse transcriptase-PCR 
approach as described previously (39). For all these mRNAs, except for Nos3, PCR primer sequence is as reported previously $(12,39,40)$. Nos3 primers were designed using Primer Select software (DNAStar, Madison, WI) and NM 021838 reference sequence in NCBI. Nos3 mRNA primer sequence was 5'>3': forward: GATTCTGGCAAGACCGATTACACGAC; reverse: CCGCGGCC AGCTCTGTCC, to amplify $228 \mathrm{bp}$. In addition to the above mRNA markers, HIF-1 $\alpha$, HYOU1, and VEGF mRNA were analyzed using Taqman Real-Time PCR assays (Applied Biosystems, Carlsbad, CA) and were normalized to endogenous control $\beta$-actin (ACTB).

Rationale. Analysis of the MHC phenotype provides important insights on the adaptation of striated muscle tissue relative to contractile characteristics and economy. IGF-I is a powerful regulator of tissue growth. Modulation of this system is accomplished both via changes in IGF-1 expression and its bioavailability as determined, in part, by the IGF binding protein family. Myostatin is a powerful negative regulator of muscle growth. Muscle ring finger-1 and Atrogin are muscle-specific E3 ligases that can modulate muscle size and adaptation via the targeting of proteins for proteolysis. Cyclin D1 is a key regulator of entry into the cell cycle. Nos3 is important for the generation of NO. In muscle, NO signaling is implicated in a number of processes including angiogenesis. VEGF is also a critical regulator of angiogenesis that functions in conjunction with $\mathrm{Hx}$-sensitive proteins such as HIF- $1 \alpha$ and HYOU1.

\section{MicroRNA Analysis}

TaqMan assays were carried out on miR-1, miR-133a, and miR206. Reverse transcriptase reactions were carried out using the TaqMan microRNA Reverse Transcription Kit (Applied Biosystems) according to the manufacturer's instructions. Real-time PCR analysis was performed with the Applied Biosystems 7900HT Sequence Detection System using TaqMan Universal PCR Master Mix and Assays-onDemand microRNA probes (Applied Biosystems). All reactions were run in duplicate. The cycle threshold for each sample was determined using SDS software version 2.3 (Applied Biosystems) and was normalized to endogenous control U6 snRNA.

\section{Statistical Analysis}

Between-group analysis was conducted using a one-way ANOVA with Bonferroni's multiple-comparison post-test using Prism software (GraphPad, La Jolla, CA). Graphical representations of the data include $95 \%$ confidence limits. Tabular data include mean \pm SE. For all statistical tests, the significance level was set at 0.05 .

\section{SUPPLEMENTARY MATERIAL}

Supplementary material is linked to the online version of the paper at http:// www.nature.com/pr

\section{ACKNOWLEDGMENTS}

The authors thank Cherryl Nugas, Ming Zeng, and Paul Bodell for their technical assistance.

\section{STATEMENT OF FINANCIAL SUPPORT}

This work was supported in part by National Institutes of Health $(\mathrm{NIH}$, Bethesda, MD) grant 2P01HD048721-06, and the University of California Irvine Institute for Clinical and Translational Science, Irvine, CA (NIH, UL1 TR000153).

Disclosure: The authors certify that there are no potential perceived conflicts of interest or financial disclosures related to this work.

\section{REFERENCES}

1. Petry CJ, Ozanne SE, Hales CN. Programming of intermediary metabolism. Mol Cell Endocrinol 2001;185:81-91.

2. Esquer C, Claure N, D'Ugard C, Wada Y, Bancalari E. Role of abdominal muscles activity on duration and severity of hypoxemia episodes in mechanically ventilated preterm infants. Neonatology 2007;92:182-6.

3. Rogers LK, Velten M. Maternal inflammation, growth retardation, and preterm birth: insights into adult cardiovascular disease. Life Sci 2011;89: $417-21$.
4. Eliakim A, Nemet D. Osteopenia of prematurity - the role of exercise in prevention and treatment. Pediatr Endocrinol Rev 2005;2:675-82.

5. Rohlicek CV, Viau S, Trieu P, Hébert TE. Effects of neonatal hypoxia in the rat on inotropic stimulation of the adult heart. Cardiovasc Res 2005;65:861-8.

6. Williams AH, Liu N, van Rooij E, Olson EN. MicroRNA control of muscle development and disease. Curr Opin Cell Biol 2009;21:461-9.

7. Scheett TP, Mills PJ, Ziegler MG, Stoppani J, Cooper DM. Effect of exercise on cytokines and growth mediators in prepubertal children. Pediatr Res 1999;46:429-34.

8. Frost RA, Lang CH. Regulation of muscle growth by pathogen-associated molecules. J Anim Sci 2008;86:Suppl 14:E84-93.

9. Han YS, Geiger PC, Cody MJ, Macken RL, Sieck GC. ATP consumption rate per cross bridge depends on myosin heavy chain isoform. J Appl Physiol 2003;94:2188-96.

10. Hoier B, Nordsborg N, Andersen S, et al. Pro- and anti-angiogenic factors in human skeletal muscle in response to acute exercise and training. J Physiol (Lond) 2012;590(Pt 3):595-606.

11. Glass DJ. PI3 kinase regulation of skeletal muscle hypertrophy and atrophy. Curr Top Microbiol Immunol 2010;346:267-78.

12. Bodell PW, Kodesh E, Haddad F, Zaldivar FP, Cooper DM, Adams GR. Skeletal muscle growth in young rats is inhibited by chronic exposure to IL-6 but preserved by concurrent voluntary endurance exercise. J Appl Physiol 2009;106:443-53.

13. Buchowicz B, Yu T, Nance DM, Zaldivar FP, Cooper DM, Adams GR. Increased rat neonatal activity influences adult cytokine levels and relative muscle mass. Pediatr Res 2010;68:399-404.

14. Moudgil R, Michelakis ED, Archer SL. Hypoxic pulmonary vasoconstriction. J Appl Physiol 2005;98:390-403.

15. Wagner PD. The critical role of VEGF in skeletal muscle angiogenesis and blood flow. Biochem Soc Trans 2011;39:1556-9.

16. Leychenko A, Konorev E, Jijiwa M, Matter ML. Stretch-induced hypertrophy activates NFkB-mediated VEGF secretion in adult cardiomyocytes. PLOS ONE 2011;6:e29055.

17. Rabinovsky ED. The multifunctional role of IGF-1 in peripheral nerve regeneration. Neurol Res 2004;26:204-10.

18. He Z, Feng L, Zhang L, Lu Y, Xu J, Lucia A. Effects of hypoxic living and training on gene expression in an obese rat model. Med Sci Sports Exerc 2012;44:1013-20.

19. Hudlicka O, Brown MD. Postnatal growth of the heart and its blood vessels. J Vasc Res 1996;33:266-87.

20. Salih DA, Tripathi G, Holding C, et al. Insulin-like growth factor-binding protein 5 (Igfbp5) compromises survival, growth, muscle development, and fertility in mice. Proc Natl Acad Sci USA 2004;101:4314-9.

21. Russell-Jones DL, Leach RM, Ward JP, Thomas CR. Insulin-like growth factor-I gene expression is increased in the right ventricular hypertrophy induced by chronic hypoxia in the rat. J Mol Endocrinol 1993; 10:99-102.

22. Adams GR, McCue SA, Bodell PW, Zeng M, Baldwin KM. Effects of spaceflight and thyroid deficiency on hindlimb development. I. Muscle mass and IGF-I expression. J Appl Physiol 2000;88:894-903.

23. Ning Y, Schuller AG, Conover CA, Pintar JE. Insulin-like growth factor (IGF) binding protein-4 is both a positive and negative regulator of IGF activity in vivo. Mol Endocrinol 2008;22:1213-25.

24. Hayot M, Rodriguez J, Vernus B, et al. Myostatin up-regulation is associated with the skeletal muscle response to hypoxic stimuli. Mol Cell Endocrinol 2011;332:38-47.

25. Chen JF, Tao Y, Li J, et al. microRNA-1 and microRNA-206 regulate skeletal muscle satellite cell proliferation and differentiation by repressing Pax7. J Cell Biol 2010;190:867-79.

26. Liu $\mathrm{H}$, Chen SE, Jin B, et al. TIMP3: a physiological regulator of adult myogenesis. J Cell Sci 2010;123(Pt 17):2914-21.

27. Rohini A, Agrawal N, Koyani CN, Singh R. Molecular targets and regulators of cardiac hypertrophy. Pharmacol Res 2010;61:269-80.

28. Del Duca D, Wong G, Trieu P, et al. Association of neonatal hypoxia with lasting changes in left ventricular gene expression: an animal model. J Thorac Cardiovasc Surg 2009;138:538-46, 546.e1. 


\section{Articles $\mid$ Radom-Aizik et al.}

29. Del Duca D, Tadevosyan A, Karbassi F, et al. Hypoxia in early life is associated with lasting changes in left ventricular structure and function at maturity in the rat. Int J Cardiol 2012;156:165-73.

30. Gonzalez NC, Wood JG. Alveolar hypoxia-induced systemic inflammation: what low PO(2) does and does not do. Adv Exp Med Biol 2010;662:27-32.

31. Dix R, Orth T, Allen J, Wood JG, Gonzalez NC. Activation of mast cells by systemic hypoxia, but not by local hypoxia, mediates increased leukocyteendothelial adherence in cremaster venules. J Appl Physiol 2003;95:2495502.

32. Gonzalez NC, Allen J, Blanco VG, Schmidt EJ, van Rooijen N, Wood JG. Alveolar macrophages are necessary for the systemic inflammation of acute alveolar hypoxia. J Appl Physiol 2007;103:1386-94.

33. Saini A, Al-Shanti N, Nasser AS, Stewart CE. Waste management cytokines, growth factors and cachexia. Cytokine Growth Factor Rev 2006;17:475-86.

34. Barry SP, Davidson SM, Townsend PA. Molecular regulation of cardiac hypertrophy. Int J Biochem Cell Biol 2008;40:2023-39.
35. Tamura DY, Moore EE, Partrick DA, Johnson JL, Offner PJ, Silliman CC. Acute hypoxemia in humans enhances the neutrophil inflammatory response. Shock 2002;17:269-73.

36. Aggoun Y. Obesity, metabolic syndrome, and cardiovascular disease. Pediatr Res 2007;61:653-9.

37. Naeije R, Hallemans R, Melot C, et al. Eicosanoids and hypoxic pulmonary vasoconstriction in normal man. Bull Eur Physiopathol Respir 1987;23:613-7.

38. Agnish ND, Keller KA. The rationale for culling of rodent litters. Fundam Appl Toxicol 1997;38:2-6.

39. Adams GR, Haddad F, Bodell PW, Tran PD, Baldwin KM. Combined isometric, concentric, and eccentric resistance exercise prevents unloading-induced muscle atrophy in rats. J Appl Physiol 2007;103: 1644-54.

40. Haddad F, Qin AX, Bodell PW, Jiang W, Giger JM, Baldwin KM. Intergenic transcription and developmental regulation of cardiac myosin heavy chain genes. Am J Physiol Heart Circ Physiol 2008;294:H29-40. 